ARTICLE

Received 8 Dec 2013 | Accepted 31 Jan 2014 | Published 26 Feb 2014 DOl: 10.1038/ncomms4368

\title{
Regulation of MKL1 via actin cytoskeleton dynamics drives adipocyte differentiation
}

Hiroyuki Nobusue ${ }^{1,2}$, Nobuyuki Onishi², Takatsune Shimizu2,3, Eiji Sugihara2,3, Yoshinao Oki ${ }^{1}$, Yuko Sumikawa1, Tatsuyuki Chiyoda ${ }^{2}$, Koichi Akashi ${ }^{4}$, Hideyuki Saya ${ }^{2,3}$ \& Koichiro Kano ${ }^{1}$

Cellular differentiation is regulated through activation and repression of defined transcription factors. A hallmark of differentiation is a pronounced change in cell shape, which is determined by dynamics of the actin cytoskeleton. Here we show that regulation of the transcriptional coactivator MKL1 (megakaryoblastic leukemia 1) by actin cytoskeleton dynamics drives adipocyte differentiation mediated by peroxisome proliferator-activated receptor $\gamma$ (PPAR $\gamma$ ), a master transcriptional regulator of adipogenesis. Induction of adipocyte differentiation results in disruption of actin stress fibres through downregulation of RhoA-ROCK signalling. The consequent rapid increase in monomeric G-actin leads to the interaction of G-actin with MKL1, which prevents nuclear translocation of MKL1 and allows expression of PPAR $\gamma$ followed by adipogenic differentiation. Moreover, we found that MKL1 and PPAR $\gamma$ act in a mutually antagonistic manner in the adipocytic differentiation programme. Our findings thus provide new mechanistic insight into the relation between the dynamics of cell shape and transcriptional regulation during cellular differentiation.

\footnotetext{
${ }^{1}$ Laboratory of Cell and Tissue Biology, College of Bioresource Sciences, Nihon University, Fujisawa, Kanagawa 252-0880, Japan. ${ }^{2}$ Division of Gene Regulation, Institute for Advanced Medical Research, Keio University School of Medicine, Tokyo 160-8582, Japan. ${ }^{3}$ Japan Science and Technology Agency, Core Research for Evolutional Science and Technology (CREST), Tokyo 102-0076, Japan. ${ }^{4}$ Department of Medicine and Biosystemic Science, Kyushu University Graduate School of Medical Science, Fukuoka 812-8582, Japan. Correspondence and requests for materials should be addressed to H.S. (email: hsaya@a5.keio.jp) or to K.K. (email: kano.kouichirou@nihon-u.ac.jp).
} 
A ctivation and repression of defined transcription factors are essential for the commitment of progenitors to a specific differentiation lineage, setting the stage for a gene expression pattern characteristic of each mature cell type ${ }^{1-3}$. Adipocyte differentiation is regulated by multiple transcription factors, with PPAR $\gamma$ and members of the CCAAT/enhancerbinding protein $(\mathrm{C} / \mathrm{EBP})$ family having central roles. Cooperative interactions among these transcription factors drive the expression of downstream target genes that are necessary for the generation and maintenance of adipocyte characteristics such as lipid accumulation and insulin sensitivity ${ }^{4}$. The key role of PPAR $\gamma$ in the commitment of mesenchymal precursors to the adipocytic differentiation programme is evident from the observation that forced expression of this protein stimulates adipogenesis in nonadipogenic fibroblastic cell lines, such as NIH 3T3 and 3T3-C2 (refs 5,6). PPAR $\gamma$ is thus thought to function as an adipocyte-specific master switch in acquisition of the adipocytic phenotype ${ }^{7}$.

The adipocytic differentiation of fibroblastic preadipocytes is accompanied by the adoption of a rounded morphology that is characteristic of mature adipocytes and allows for maximal lipid storage $^{8}$. Cell morphology is determined primarily by the actin cytoskeleton ${ }^{9}$. Adipocyte differentiation is thus associated with a shift in the structures formed by filamentous (F) actin from stress fibres to cortical fibres ${ }^{10,11}$. The relation between such reorganization of the actin cytoskeleton and the PPAR $\gamma$-mediated adipocytic differentiation programme has remained unclear, however.

We have previously established a preadipocyte cell line derived from mouse dedifferentiated fat (DFAT) cells and which possesses a higher potential to differentiate into adipocytes compared with the mouse preadipocyte cell line 3T3-L1 (ref. 12). Unlike 3T3-L1 cells, these DFAT cells do not undergo spontaneous adipogenesis, with the adipocytic differentiation of DFAT cells thus being more tightly controlled than that of 3T3-L1 cells ${ }^{12,13}$. Here, we examine the roles of actin cytoskeleton remodelling in adipocytic differentiation by using DFAT cells. We show that the rapid disruption of actin stress fibres through downregulation of RhoA-ROCK signalling and the consequent increase in monomeric G-actin levels are observed before adipocyte differentiation. We also found that interaction of monomeric G-actin with the transcriptional coactivator MKL1 is the key event for initiating PPAR $\gamma$ expression. Moreover, MKL1 and PPAR $\gamma$ were shown to act in a mutually antagonistic manner in the adipocytic differentiation programme. Our findings provide a new insight into the regulatory mechanism of adipocyte differentiation.

\section{Results}

Adipogenesis requires disruption of actin stress fibres. We first examined the changes in the actin cytoskeleton and PPAR $\gamma$ expression during adipogenesis with the use of an established DFAT cell line. Before induction of adipogenesis, DFAT cells manifest a fibroblastic morphology with well-developed actin stress fibres (Fig. 1a). At $24 \mathrm{~h}$ after the induction of adipocytic differentiation with the combination of 3-isobutyl-1-methylxanthine (IBMX), dexamethasone and insulin, the cells exhibit a well-spread morphology associated with the disruption of most stress fibres (Fig. 1a,c; Supplementary Movie 1). The upregulation of PPAR $\gamma$ expression at both mRNA and protein levels is evident at $48 \mathrm{~h}$ (Fig. 1a,b), at which time the cell morphology has become stellate and the cortical actin structures characteristic of adipocytes are evident (Fig. 1a). The expression of perilipin (a marker of terminal adipocyte differentiation) and the accumulation of lipid droplets are observed from $60 \mathrm{~h}$, at which time the cell morphology has become more rounded (Fig. 1a). Together, these results thus suggested that disruption of actin stress fibres precedes the induction of PPAR $\gamma$ expression during adipocyte differentiation.

Previous studies have shown that actin cytoskeleton dynamics associated with cell rounding in response to mechanical or physical stimuli (such as matrix stiffness and cytoskeletal tension) result in adipogenic differentiation of 3T3-F442A preadipocytes or mesenchymal stem cells by a mechanism that involves RhoA-ROCK (Rho-kinase) signalling ${ }^{14-18}$, which regulates the formation of actin stress fibres and focal adhesions ${ }^{19,20}$. On the other hand, ectopic expression of PPAR $\gamma$ in mesenchymal stem cells or NIH 3T3 fibroblasts has been found to induce not only expression of adipose-specific genes but also morphological differentiation, including the accumulation of triglyceride droplets $5,21-24$. It has remained unknown, however, whether the pathways underlying the induction of adipogenesis either by PPAR $\gamma$ or by changes in cell shape operate independently of each other or in an interdependent manner. To address this question, we tested the effects both of RNA interference (RNAi)-mediated depletion of cofilin1, a protein that promotes the disassembly of actin filaments ${ }^{25}$, and of phalloidin (a cytoskeletal fixative) on PPAR $\gamma$ expression during adipocyte differentiation. Either knockdown of cofilin1 with specific small interfering RNAs (siRNAs) or treatment with phalloidin blocked the disruption of actin stress fibres as well as markedly inhibited both the upregulation of PPAR $\gamma$ and PPAR $\gamma$ target gene expression and the accumulation of lipid droplets elicited by inducers of adipocyte differentiation in DFAT cells and 3T3-L1 preadipocytes (Fig. 1d,e; Supplementary Fig. 1). These data thus indicated that the disruption of actin stress fibres is required

Figure 1 | Disruption of actin stress fibres is required for adipocyte differentiation. (a) Fluorescence microscopy of the actin cytoskeleton (stained with phalloidin) as well as of PPAR $\gamma$ or perilipin expression during adipogenesis in DFAT cells. Nuclei were stained with Hoechst 33342 (blue fluorescence). Scale bars, $20 \mu \mathrm{m}$. (b) Relative abundance of Pparg mRNA during adipocytic differentiation in DFAT cells (left panel). (a-f) $P<0.05$, Tukey's honest significant difference test. A nuclear fraction prepared from the cells was also subjected to immunoblot analysis of PPAR $\gamma$ and lamin C (loading control) (right panel). (c) Time-lapse imaging of DFAT cells expressing red fluorescent protein (RFP)-tagged actin at the indicated times (hours:minutes). Scale bars, $50 \mu \mathrm{m}$. (d) 3T3-L1 preadipocytes transfected with cofilin1 (siCf17-a) or control (siControl) siRNAs were exposed to inducers of differentiation for $96 \mathrm{~h}$. They were then subjected to fluorescence microscopic analysis of the actin cytoskeleton and PPAR $\gamma$ expression. Nuclei were stained with Hoechst 33342. Scale bars, $50 \mu \mathrm{m}$. Relative abundance of Pparg, Cebpa, Fabp4, Slc2a4 and Plin1 mRNAs. ${ }^{\star} P<0.05$, Student's t-test. (e) Oil red O (ORO) staining of cells treated as in d. Bars, $100 \mu \mathrm{m}$. The absorbance at $510 \mathrm{~nm}\left(A_{510}\right)$ of dye extracted from the stained cells was also determined. ${ }^{*} P<0.05$, Student's $t$-test. (f) Immunoblot analysis of active and total forms of Rho proteins during adipocyte differentiation in DFAT cells (left panels). GAPDH was examined as a loading control. Quantification of the immunoblotting data was performed using densitometry. Data were normalized to the amount of total Rho (right panel). ${ }^{\star} P<0.05$, Student's $t$-test. n.s., not significant. (g) Fluorescence microscopy of the actin cytoskeleton as well as of PPAR $\gamma$ or perilipin expression in DFAT cells expressing HA-tagged RhoAV14 or RhoAN19 and exposed for $96 \mathrm{~h}$ to inducers of differentiation in the absence or presence of $\mathrm{Y}-27632(30 \mu \mathrm{M})$ or CytD $(0.2 \mu \mathrm{M})$. Nuclei were stained with Hoechst 33342. Scale bars, $20 \mu \mathrm{m}$. At least 50 HA-positive cells were scored for determination of the percentage of those expressing PPAR $\gamma .{ }^{\star} P<0.05$, Student's $t$-test. All quantitative data are means $\pm s . d$. ( $n=3$ experiments fibres). 
for the induction of PPAR $\gamma$ expression during adipocyte differentiation.

RhoA-ROCK signalling regulates adipogenesis. We next investigated whether RhoA-ROCK signalling plays a role in adipogenic differentiation dependent on the disruption of actin stress fibres in DFAT cells. The GTP-bound (active) form of Rho proteins was detected at a high level before adipogenic induction and was markedly reduced in abundance within $24 \mathrm{~h}$ after exposure of the cells to inducers of adipocyte differentiation a
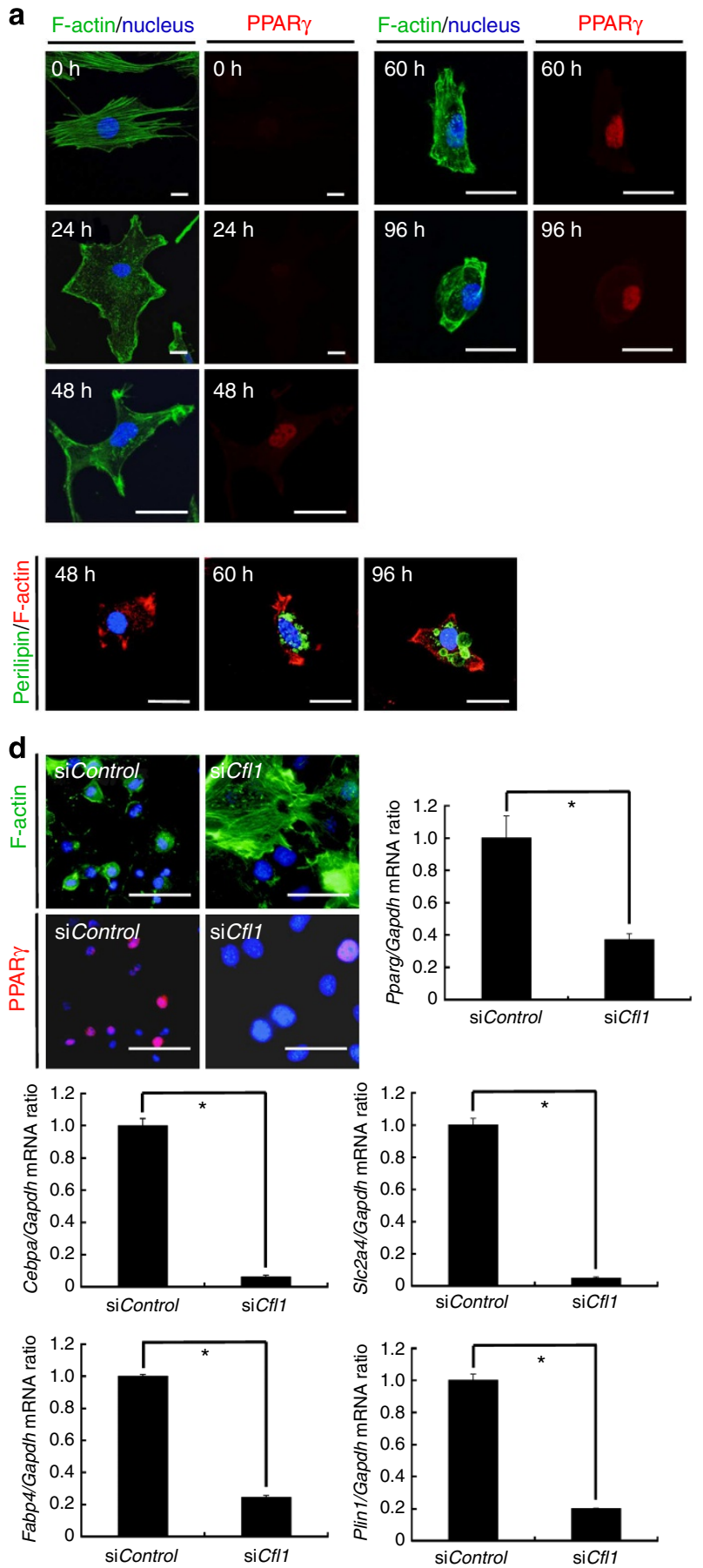

e
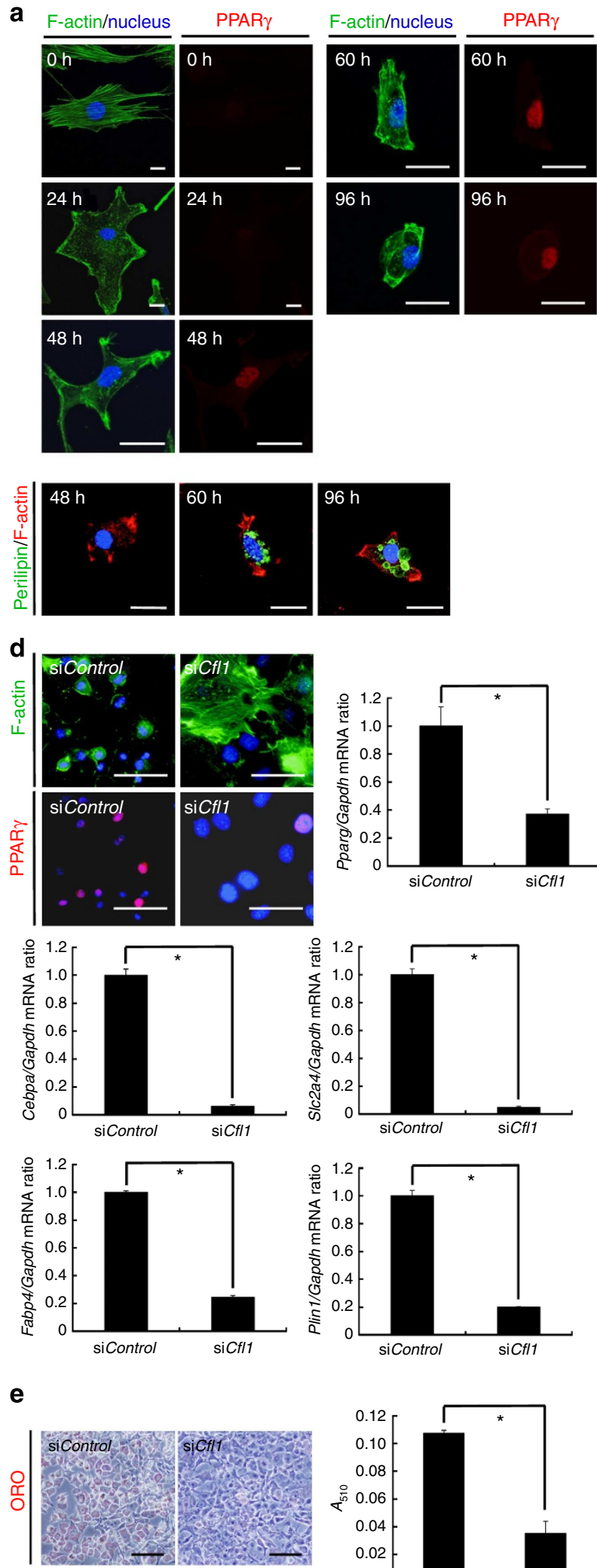
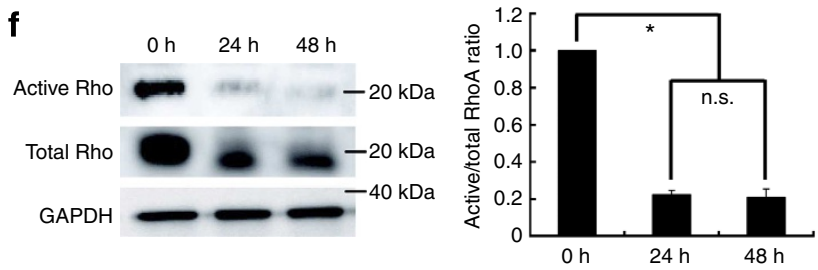

g
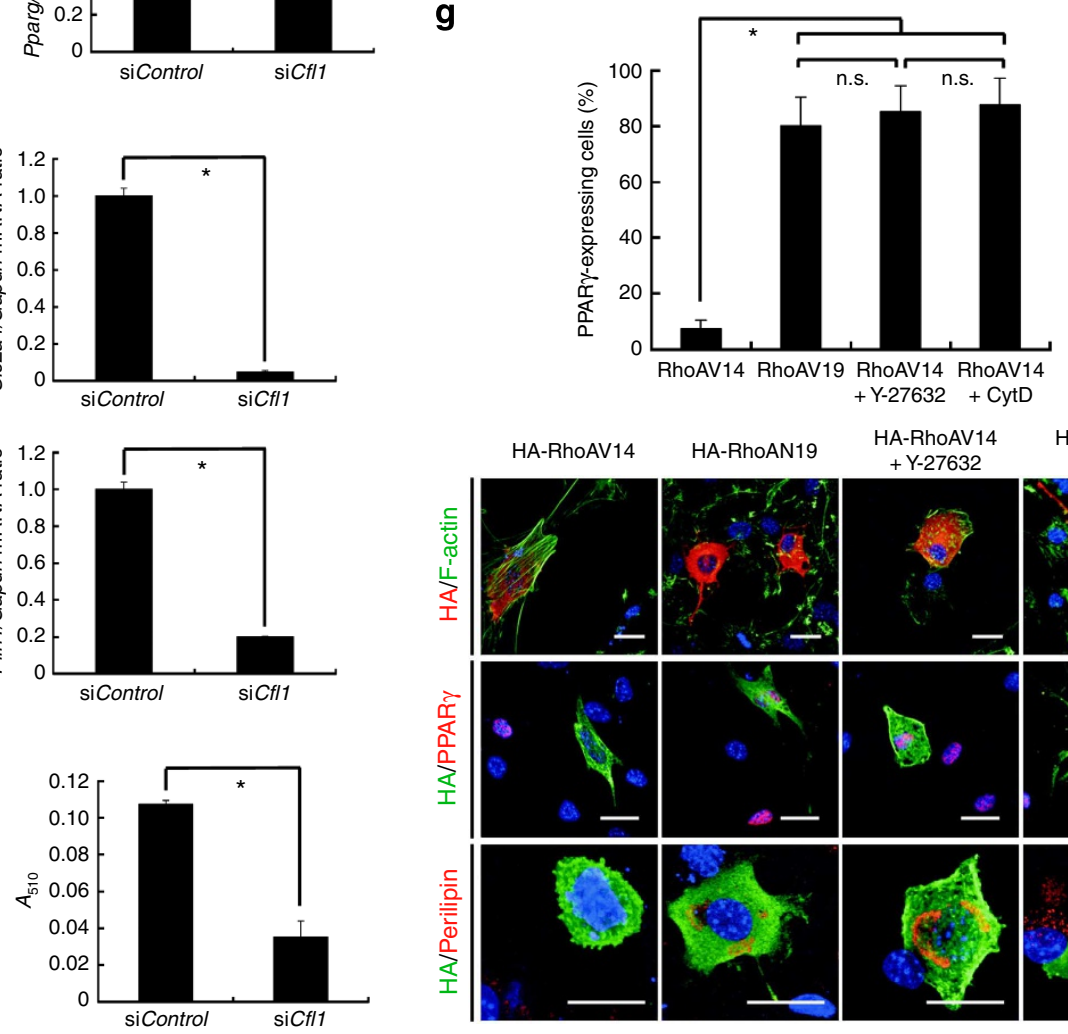

b

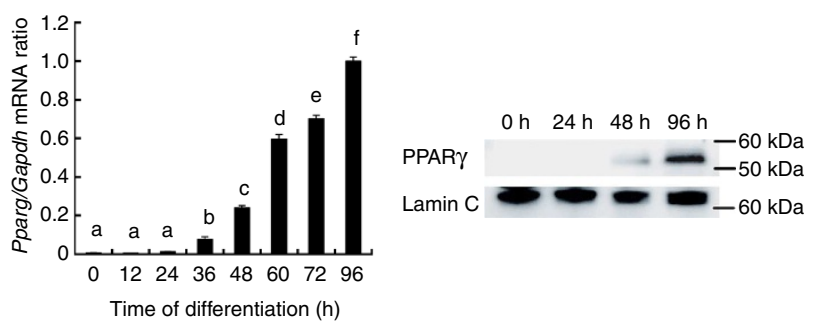

C

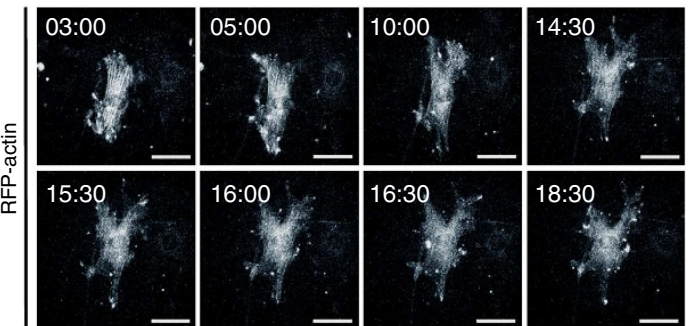

HA-RhoAV14

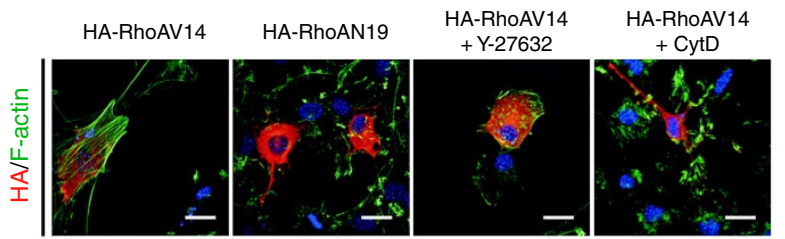

文
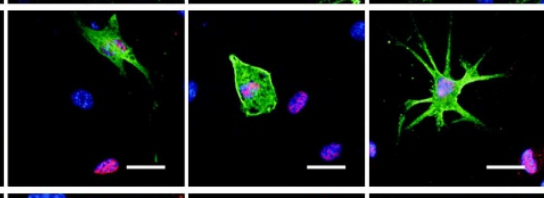

ㄴ.

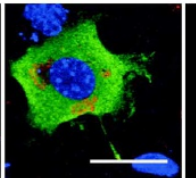


(Fig. 1f). We transiently transfected DFAT cells with plasmids encoding hemagglutinin epitope (HA)-tagged dominant active (RhoAV14) or dominant negative (RhoAN19) mutants of RhoA and then exposed the cells to inducers of adipocytic differentiation for $96 \mathrm{~h}$. Expression of RhoAV14 inhibited both remodelling of the actin cytoskeleton as well as the induction of PPAR $\gamma$ and perilipin expression, and these inhibitory effects were prevented by treatment with the ROCK inhibitor Y-27632 (Fig. 1g). We also determined whether the effects of ROCK inhibition in RhoAV14expressing cells were mimicked by the actin-depolymerizing agent cytochalasin $\mathrm{D}$ (CytD). The addition of CytD indeed restored remodelling of the actin cytoskeleton as well as the expression of PPAR $\gamma$ and perilipin in RhoAV14-expressing cells to levels similar to those apparent in RhoAV14-expressing cells treated with Y-27632 or in cells expressing RhoAN19 (Fig. 1g). These results thus indicated that RhoA-ROCK signalling regulates adipocyte differentiation through control of remodelling of the actin cytoskeleton.

G-actin induces adipogenesis by controling MKL1 localization. Recent studies have identified mechanisms by which actin dynamics directly affect gene transcription ${ }^{26}$. Monomeric G-actin binds to the transcriptional coactivator MKL1 (also known as MAL or MRTF-A) and prevents it from translocating to the nucleus and activating transcription ${ }^{27,28}$. Furthermore, in multipotent mesenchymal stem cells, Med23 functions as a molecular switch between ELK1 and MKL1 that controls differentiation into adipocytes or smooth muscle cells ${ }^{29}$, suggesting links between MKL1 and adipogenesis. To explore whether the control of MKL1 translocation by cellular G-actin contributes to the regulation of PPAR $\gamma$ expression and adipocyte differentiation, we established DFAT cells expressing mCherry fused to MKL1 (mCherry-MKL1; Supplementary Fig. 2a) and investigated G-actin levels and mCherry-MKL1 localization before and after the induction of adipocyte differentiation. F-actin was rapidly depolymerized to monomeric G-actin after the induction of adipocyte differentiation, with the increase in cellular G-actin concentration (Fig. 2a). mCherry-MKL1 was detected exclusively in the nucleus before adipogenic induction but was predominantly pancellular and/or cytoplasmic after the induction of adipogenesis (Fig. 2a). PPAR $\gamma$ expression was detected in cells having the cytoplasmic localization of mCherryMKL1 (Supplementary Fig. 2b).

We next tested whether actin-depolymerizing agents latrunculin A (LatA), which increases monomeric G-actin ${ }^{27,30}$, or swinholide A (SwinA), which increases dimeric actin that does not interact with MKL1 (refs 27,30,31), might mimic the effects of inducers of adipogenesis on dynamics of the actin cytoskeleton and MKL1 localization in DFAT cells expressing mCherry-MKL1. Indeed, LatA or SwinA induced disruption of actin stress fibres and increased the cellular abundance of G-actin monomers or dimers, respectively (Fig. 2b; Supplementary
Fig. 2c). LatA treatment caused the cytoplasmic sequestration of mCherry-MKL1, whereas SwinA treatment resulted in the nuclear localization (Fig. 2b). Furthermore, LatA alone induced a significant increase in the expression of PPAR $\gamma$ and PPAR $\gamma$ target genes unlike SwinA (Fig. 2c,d). These results suggested that monomeric G-actin formation leads to adipocyte differentiation by blocking the nuclear import of MKL1, but not by other consequences of disruption of actin stress fibres. Similarly to LatA, treatment with Y-27632, elicited disruption of actin stress fibres and the cytoplasmic sequestration of MKL1, resulting in a marked increase in the expression of PPAR $\gamma$ and PPAR $\gamma$-target genes as well as in the accumulation of lipid droplets in DFAT cells and 3T3-L1 preadipocytes (Supplementary Fig. 3).

Adipogenesis requires interaction between G-actin and MKL1. We also asked whether the interaction between G-actin and MKL1 and the consequent cytoplasmic sequestration of MKL1 contribute to adipocyte differentiation. To address this issue, we established DFAT cells and 3T3-L1 preadipocytes that express a FLAG epitope-tagged fusion protein of the oestrogen receptor (ER) and either MKL1 or a deletion mutant of MKL1 (MKL1$\mathrm{N} 100)$ that lacks the $100 \mathrm{NH}_{2}$-terminal amino acids of the full-length protein and therefore does not contain the RPEL actin-binding domain ${ }^{32,33}$ (Supplementary Fig. 4a). Exposure of these cells to the ER agonist 4-hydroxytamoxifen (TAM) induced the nuclear translocation of each fusion protein (Fig. 3a). The nuclear translocation of ER-MKL1 was accompanied by a marked decrease in the expression of PPAR $\gamma$ and PPAR $\gamma$ target genes as well as in the accumulation of lipid droplets in cells also exposed to inducers of adipocytic differentiation (Fig. 3b-d; Supplementary Fig. 4b-d). Moreover, expression of ER-MKL1N100, which fails to bind G-actin and a substantial proportion of which was localized to the nucleus even in the absence of TAM (Fig. 3b; Supplementary Fig. 4b), resulted in marked suppression of adipogenesis in the absence or presence of TAM compared with that apparent in cells expressing ER-MKL1 (Fig. 3b-d; Supplementary Fig. 4b-d). We also tested if ER-MKL1-N100 reverts the effects elicited by treatment with LatA in DFAT cells and 3T3-L1 preadipocytes. The expression of ER-MKL1-N100, which was predominantly localized to the nucleus even after treatment with LatA and remarkably impaired the expression of PPAR $\gamma$ and PPAR $\gamma$ target genes compared with that of ER-MKL1 (Fig. 3e,f; Supplementary Fig. 4e,f). These findings significantly strengthen our conclusion that the increase in the cytoplasmic abundance of G-actin triggers adipocyte differentiation via regulation of the subcellular localization of MKL1.

Loss of MKL1 drives adipocyte differentiation. We further assessed whether RNAi-mediated depletion of MKL1 alone might induce PPAR $\gamma$ expression and adipocyte differentiation in the absence of an adipogenic cocktail. In both DFAT cells and 3T3-L1

\footnotetext{
Figure 2 | The increase of G-actin accumulation resulting from disruption of actin stress fibres is involved in the cytoplasmic sequestration of MKL1 and adipocyte differentiation in DFAT cells. (a) Immunofluorescence analysis of mCherry and G-actin (stained with DNase I) in cells stably expressing mCherry-MKL1 at 0 or $48 \mathrm{~h}$ after the induction of adipogenesis(upper panels). Nuclei were stained with Hoechst 33342 . Scale bars, $100 \mu \mathrm{m}$. At least 300 cells per coverslip were scored for determination of the percentage of the subcellular localization of MKL1 (lower panel). N, nuclear; $\mathrm{N} / \mathrm{C}$, comparable intensity in nucleus and cytoplasm; C, cytoplasmic. (b) Immunofluorescence analysis of mCherry and G-actin in mCherry-MKL1expressing cells exposed to Lat $A(0.4 \mu \mathrm{M})$, Swin $A(0.1 \mu \mathrm{M})$ or dimethyl sulfoxide (DMSO, control) for $6 \mathrm{~h}$ in growth medium without an adipogenic cocktail (upper panels). Nuclei were stained with Hoechst 33342. Scale bars, $50 \mu \mathrm{m}$. Quantitation of immunofluorescence microscopy; localization of the indicated mCherry-MKL1 was scored in 300 cells as in panel a (lower panel). (c) Relative abundance of Pparg, Cebpa, Fabp4, Slc2a4, and Plin1 mRNAs in cells treated as in $\mathbf{b}$. ND, not detected. (d) Cells treated as in $\mathbf{b}$ were cultured for an additional $18 \mathrm{~h}$ (total of $24 \mathrm{~h}$ ) and then subjected to immunofluorescence analysis of mCherry and PPAR $\gamma$ expression. Nuclei were stained with Hoechst 33342. Scale bars, $50 \mu \mathrm{m}$. All quantitative data are means \pm s.d. $\left(n=3\right.$ experiments). ${ }^{\star} P<0.05$, Student's $t$-test.
} 
preadipocytes, knockdown of Mkl1 expression (Supplementary Fig. 5a) resulted in a marked increase both in the expression of PPAR $\gamma$ and PPAR $\gamma$ target genes as well as in the extent of lipid droplet accumulation (Fig. 4a; Supplementary Fig. 5b,c). Moreover, we found that depletion of MKL1 alone resulted in a marked increase in the expression of PPAR $\gamma$ and PPAR $\gamma$ target genes even in NIH 3T3 nonadipogenic fibroblasts (Fig. 4b; Supplementary Fig. 5a). These findings indicated that loss of MKL1 function elicits PPAR $\gamma$ expression and adipocyte differentiation in vitro.

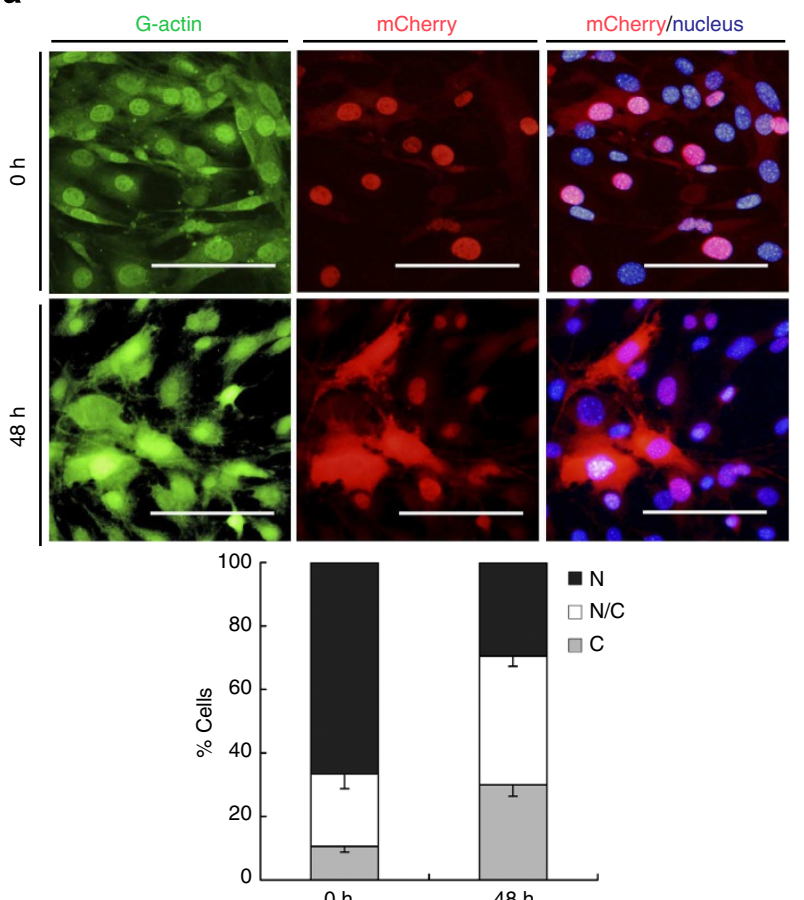

b
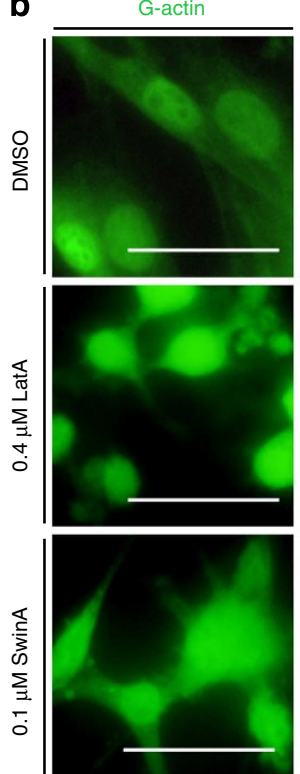

mCherry

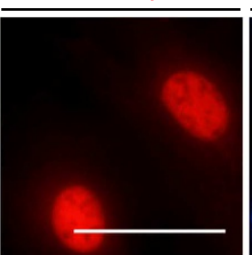

mCherry/nucleus
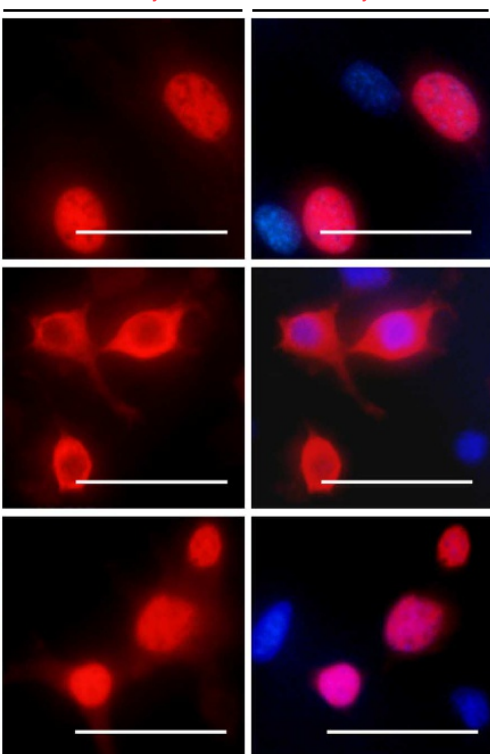

\section{C}
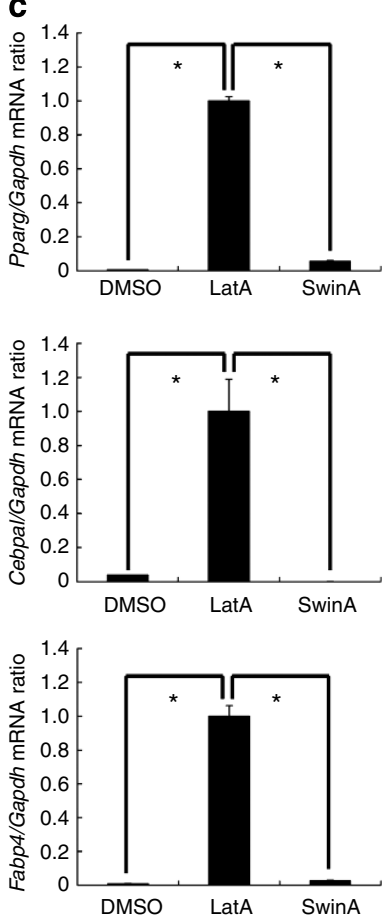

d
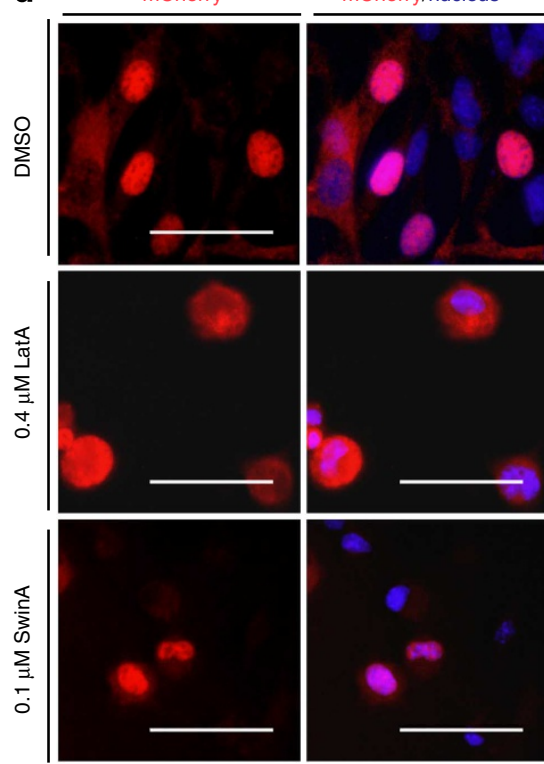

mCherry/nucleus
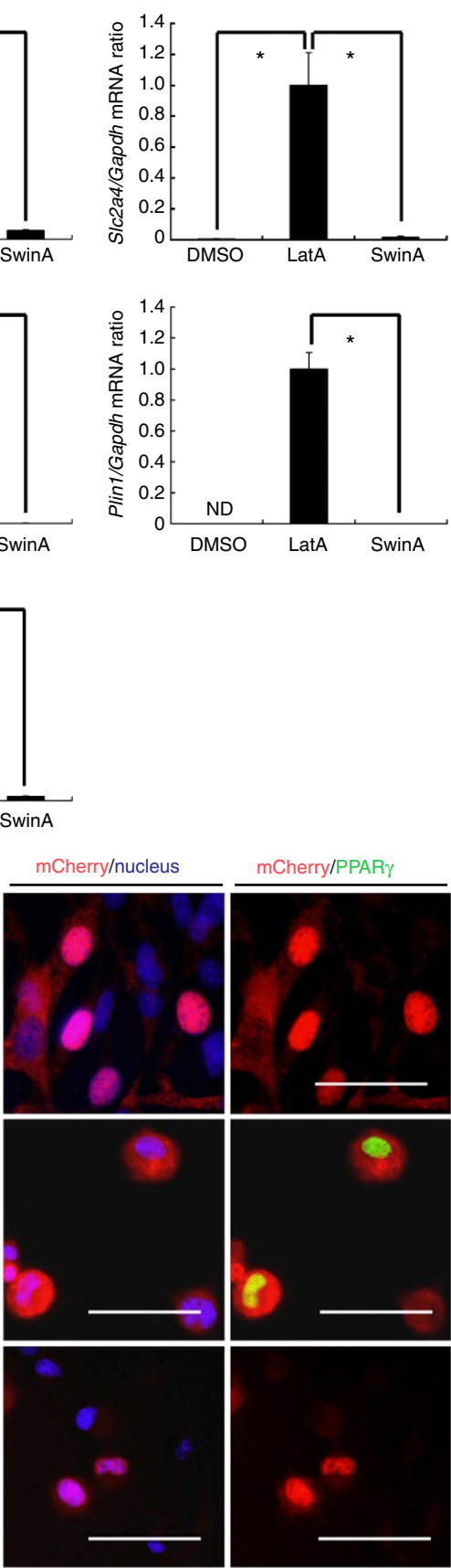

mCherry/PPAR $\gamma$
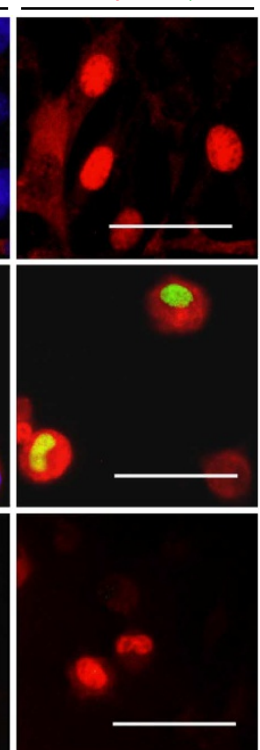

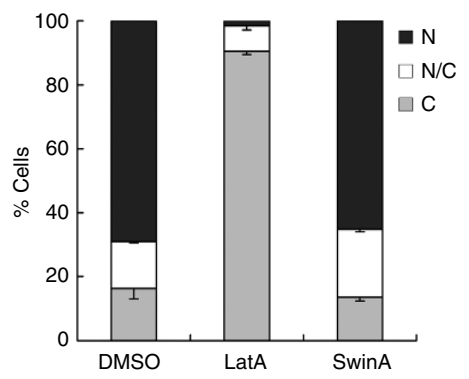


a
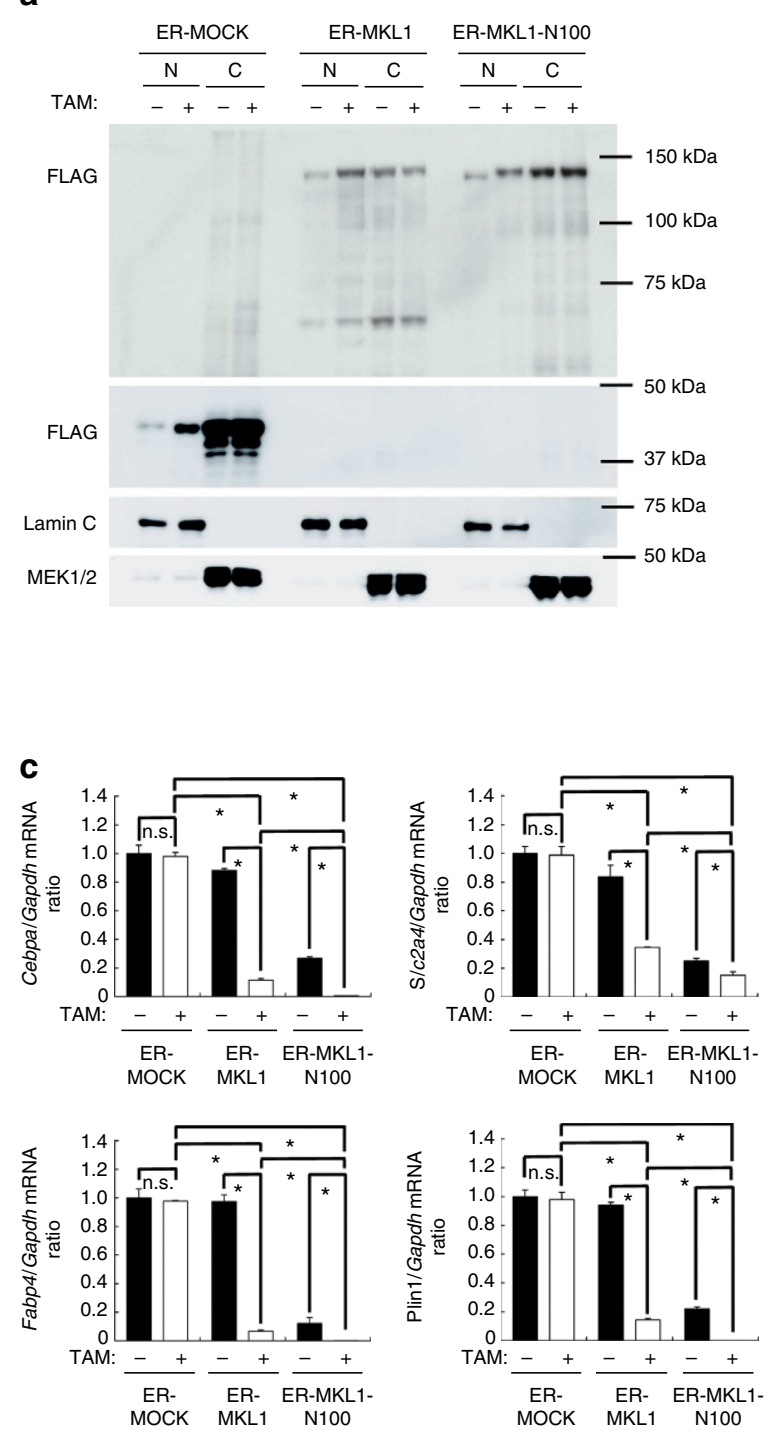

d
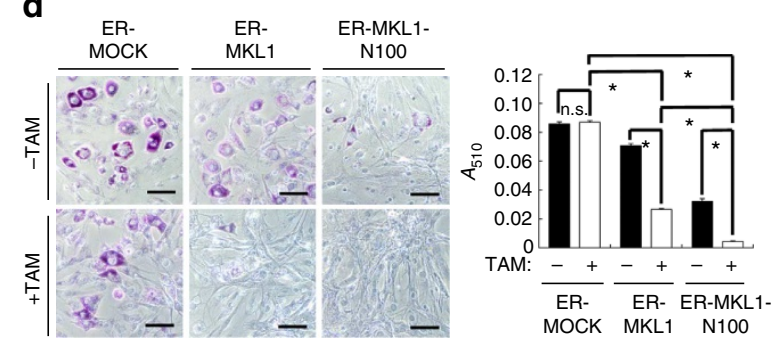

b
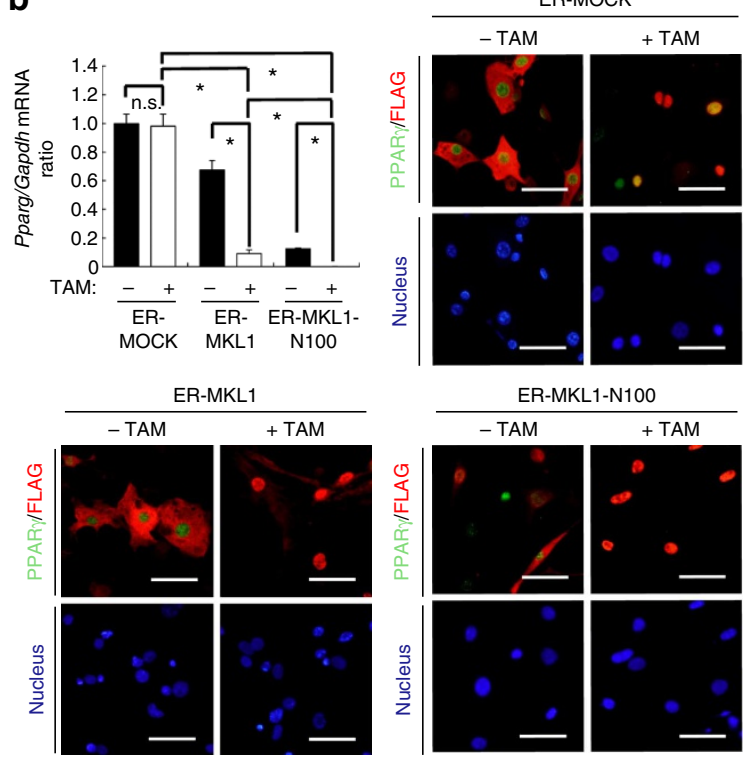

e
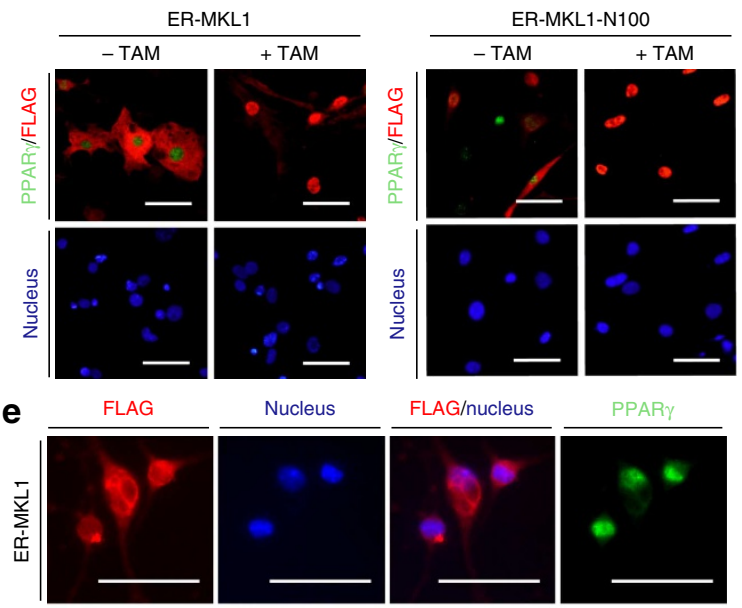

FLAG/nucleus
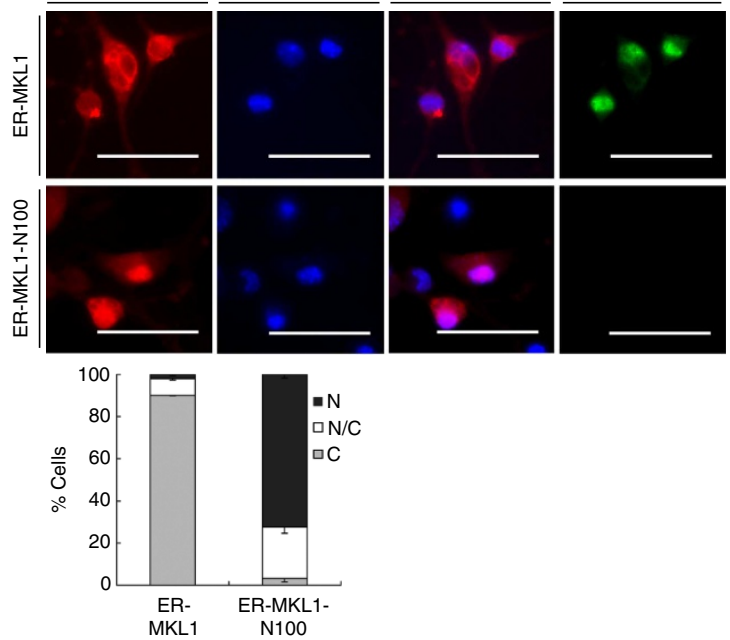

f

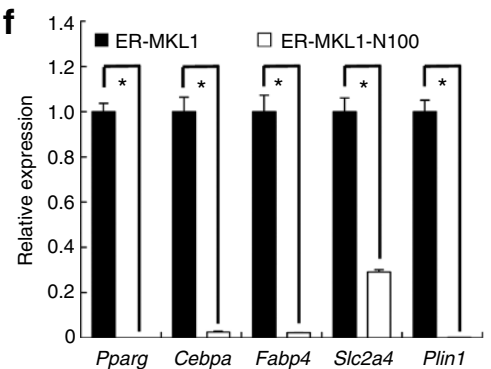

Figure 3 | Interaction between G-actin and MKL1 and the consequent cytoplasmic sequestration of MKL1 are required for adipocytic differentiation in DFAT cells. (a) Cells stably expressing $3 \times$ FLAG-tagged ER (ER-MOCK, control), ER-MKL1 or ER-MKL1-N100 were exposed for $48 \mathrm{~h}$ to inducers of adipogenic differentiation in the absence or presence of TAM $(1 \mu \mathrm{M})$, after which nuclear $(N)$ and cytoplasmic $(C)$ fractions were prepared from the cells and subjected to immunoblot analysis of FLAG, lamin C (nuclear marker) and MEK1/2 (cytoplasmic marker). (b) Cells treated as in a were analysed for the relative abundance of Pparg mRNA or subjected to immunofluorescence analysis of FLAG and PPAR $\gamma$. Nuclei were stained with Hoechst 33342. Scale bars, $50 \mu \mathrm{m}$. (c) Relative abundance of Cebpa, Fabp4, S/c2a4 and Plin1 mRNAs in cells treated as in a. (d) Cells treated as in a were cultured for an additional $48 \mathrm{~h}$ (total of $96 \mathrm{~h}$ ) and then stained with oil red O. Scale bars, $100 \mu \mathrm{m}$. The $A_{510}$ of dye extracted from the stained cells was also determined. n.s., not significant. (e) Cells stably expressing ER-MKL1 or ER-MKL1-N100 were exposed for $24 \mathrm{~h}$ to Lat A (0.4 $\mu$ M) in the presence of TAM $(1 \mu \mathrm{M})$ and then subjected to immunofluorescence analysis of FLAG and PPAR $\gamma$ (upper panels). Nuclei were stained with Hoechst 33342. Scale bars, $50 \mu \mathrm{m}$. At least 300 cells per coverslip were scored for determination of the percentage of the subcellular localization of MKL1 (centre panel). N, nuclear; N/C, comparable intensity in nucleus and cytoplasm; C, cytoplasmic. (f) Relative abundance of Pparg, Cebpa, Fabp4, Slc2a4 and Plin 1 mRNAs in cells treated as in e. All quantitative data are means \pm s.d. ( $n=3$ experiments). ${ }^{\star} P<0.05$, Student's $t$-test. 

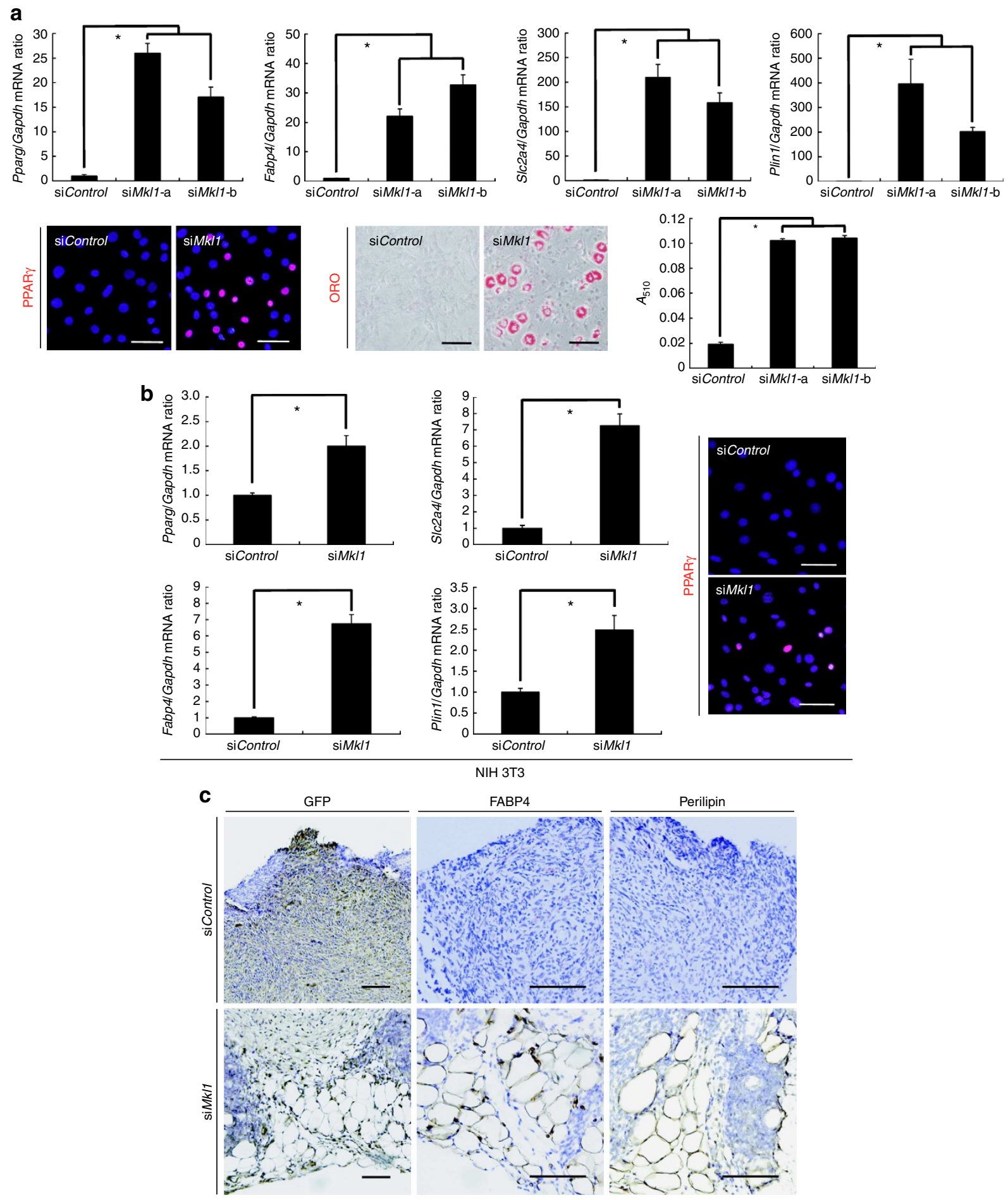

Figure 4 | Loss of MKL1 is sufficient to drive adipocyte differentiation in vitro and in vivo. (a) DFAT cells were transiently transfected with MKL1 ( $\mathrm{siMk} / 1-\mathrm{a}$ or -b) or control (siControl) siRNAs for $48 \mathrm{~h}$ and then cultured in growth medium without an adipogenic cocktail for $96 \mathrm{~h}$, after which the relative abundance of Pparg, Fabp4, Slc2a4 and Plin1 mRNAs was determined (upper panels). The cells were also subjected to immunofluorescence analysis of PPAR $\gamma$ expression (bottom left panel). Nuclei were stained with Hoechst 33342 . Scale bars, $100 \mu \mathrm{m}$. They were also stained with oil red $\mathrm{O}$ (bottom centre panel; Scale bars, $100 \mu \mathrm{m}$ ), and the $A_{510}$ of dye extracted from the stained cells was determined (bottom right panel). (b) NIH $3 T 3$ fibroblasts were transiently transfected with MKL1 (siMkl1-a) or control siRNAs for $48 \mathrm{~h}$ and then cultured in growth medium without an adipogenic cocktail for $96 \mathrm{~h}$, after which the relative abundance of Pparg, Fabp4, S/c2a4, and Plin1 mRNAs was determined (left panels). The cells were also subjected to immunofluorescence analysis of PPAR $\gamma$ expression (right panel). Nuclei were stained with Hoechst 33342. Scale bars, $100 \mu \mathrm{m}$. (c) DFAT cells were transiently transfected with MKL1 (siMkl1-a) or control siRNAs for $48 \mathrm{~h}$ and then injected subcutaneously into mice. Serial sections of the injection site were subjected to immunohistochemical staining of GFP (to identify injected cells) as well as of FABP4 and perilipin (markers of terminal adipogenic differentiation) at 2 weeks after injection. The sections were counterstained with hematoxylin. Data are representative of five mice per group. Scale bars, $100 \mu \mathrm{m}$. All quantitative data are means \pm s.d. ( $n=3$ experiments). ${ }^{\star} P<0.05$, Student's $t$-test. 
To investigate the function of MKL1 in adipocyte differentiation in vivo, we transfected DFAT cells (which are derived from green fluorescent protein (GFP) transgenic mice) ${ }^{12}$ with MKL1 siRNA for $48 \mathrm{~h}$ (resulting in a significant decrease in $M k l 1$ expression but no change in Pparg expression at this time (Supplementary Fig. 5d)) and then injected the cells subcutaneously into mice. At 2 weeks after injection, many fully differentiated adipocytes were observed at the injection site in the animals that received cells transfected with MKL1 siRNA but not in those that received cells transfected with a control siRNA (Fig. 4c). The transplanted MKL1-depleted cells were thus strongly positive for markers of terminal adipogenic differentiation (FABP4 and perilipin) as well as for GFP (Fig. 4c). Together, these data revealed that MKL1 functions as a gatekeeper that controls adipocyte differentiation both in vitro and in vivo. It was previously reported that the postpartum mammary glands of Mkl1 knockout female mouse show premature involution and markedly increased white adipose tissue $^{34}$. This phenotype of Mkl1 knockout mouse is concordant with our finding that loss of Mkl1 promotes adipocyte differentiation programme.

We also performed microarray analysis to examine the gene expression profile of cells treated with ROCK inhibitor (Y-27632) or depleted MKL1 expression by siRNA (siMkl1) and functional classification of highly expressed genes ( $>2$-fold). The gene ontology (GO) analysis revealed the most significant biological function was 'fat cell differentiation' (GO:0045444) in both ROCK inhibition and MKL1 knockdown (Table 1). Furthermore, hierarchical clustering of all samples for 133 fat cell differentiation-associated genes demonstrated an extremely high similarity of the gene expression pattern between Y-27632 sample and siMkl1 sample (Supplementary Fig. 6a), suggesting that either ROCK inhibition or MKL1 knockdown alone predominantly induces the expression of adipocyte differentiation-associated genes in global gene expression level. Furthermore, we extracted the expression patterns of a large number of well-documented serum response factor (SRF)-MKL1 target genes ${ }^{26}$ from those microarray data. The relative expression of these SRF-MKL1 target genes tends to decrease on ROCK inhibition and MKL1 knockdown (Supplementary Fig. 6b), suggesting that either ROCK inhibition or MKL1 knockdown in common suppresses the expression of genes downstream of MKL1.

\begin{tabular}{|c|c|c|}
\hline $\begin{array}{l}\text { Gene Ontology class (Biological process } \\
\text { with GO ID) }\end{array}$ & $\begin{array}{l}\text { No. of } \\
\text { genes } \\
\text { in class }\end{array}$ & $\begin{array}{l}\text { B-H } \\
\text { P-value }\end{array}$ \\
\hline \multicolumn{3}{|l|}{ Y-27632>DMSO (160 genes) } \\
\hline GO:0045444-fat cell differentiation & 14 & $1.57 \mathrm{E}-10$ \\
\hline GO:0050873-brown fat cell differentiation & 10 & $1.31 \mathrm{E}-08$ \\
\hline GO:0050896-response to stimulus & 58 & $4.69 \mathrm{E}-06$ \\
\hline GO:0006629-lipid metabolic process & 26 & $7.94 \mathrm{E}-05$ \\
\hline GO:0002526-acute inflammatory response & 10 & $8.33 \mathrm{E}-05$ \\
\hline \multicolumn{3}{|l|}{ siMkl1> siControl (213 genes) } \\
\hline GO:0045444-fat cell differentiation & 19 & $7.26 \mathrm{E}-16$ \\
\hline GO:0050873-brown fat cell differentiation & 15 & $8.58 \mathrm{E}-16$ \\
\hline GO:0006629-lipid metabolic process & 33 & $6.41 \mathrm{E}-06$ \\
\hline GO:0044255—cellular lipid metabolic process & 27 & $5.40 \mathrm{E}-06$ \\
\hline GO:0002526-response to hormone stimulus & 16 & $7.75 \mathrm{E}-06$ \\
\hline
\end{tabular}

GO analysis were performed individually on the highly expressed genes ( $>2$-fold) in Y-27632 sample (160 genes) or in siMkl1 sample (213 genes). All functional categories demonstrated enhanced statistical representation.
PPAR $\gamma$ induces $M k l 1$ downregulation during adipogenesis. We noticed that treatment with Y-27632, which elicited remodelling of the actin cytoskeleton, not only prevented nuclear translocation of MKL1 but also induced a significant decrease in the amount of Mkl1 mRNA in DFAT cells and 3T3-L1 preadipocytes (Supplementary Fig. 3i). Moreover, we found that Mkl1 expression correlated inversely with Pparg expression during adipocytic differentiation (Fig. 5a). To test whether PPAR $\gamma$ contributes to Mkl1 downregulation during adipogenesis, we investigated the effect of PPAR $\gamma$ knockdown on Mkl1 expression. We established DFAT cells stably depleted of PPAR $\gamma$ as a result of retrovirusmediated expression of a specific short hairpin RNA (shRNA) (Fig. 5b). Although control cells expressing a luciferase shRNA manifested a significant decrease in the abundance of Mkl1 mRNA after exposure to inducers of adipocytic differentiation, cells expressing the PPAR $\gamma$ shRNA did not (Fig. 5b). To clarify further the influence of PPAR $\gamma$ on $M k l 1$ expression, we generated DFAT cells that stably overexpress PPAR $\gamma$ (Fig. 5c). Overexpression of PPAR $\gamma$ resulted in a significant decrease in the amount of Mkl1 mRNA (Fig. 5c), indicating that PPAR $\gamma$ indeed suppresses the expression of $M k l 1$, resulting in the continuous activation of PPAR $\gamma$ required for the completion of adipocyte differentiation.

\section{Discussion}

On the basis of our observations, we propose a model for the control of adipocyte differentiation in which regulation of MKL1 by disruption of actin stress fibres triggers the differentiation programme (Fig. 5d). Exposure of preadipocytes to an adipogenic cocktail results in the rapid disruption of actin stress fibres as a consequence of downregulation of RhoA-ROCK signalling. The resulting increase in the amount of G-actin leads to the binding of G-actin to MKL1 and to inhibition of the nuclear translocation and transcriptional regulatory activity of the latter protein. Transcription of the Pparg gene is thereby activated, and the encoded protein then mediates not only upregulation of the expression of genes (such as Fabp4, Plin and Slc2a4) associated with the adipocyte phenotype but also downregulation of $M k l 1$ expression to allow completion of adipogenesis.

Several preadipocyte cell lines undergo differentiation on treatment with a standard adipogenic cocktail consisting of IBMX, dexamethasone and insulin ${ }^{35}$. Both IBMX and dexamethasone trigger the activation of protein kinase A by increasing the intracellular concentration of cAMP, thereby promoting adipocyte differentiation ${ }^{36}$. The major role of protein kinase $\mathrm{A}$ in adipogenesis has been thought to lie in downregulation of RhoA and ROCK activity ${ }^{37}$. In the present study, we found that Rho activity is rapidly reduced after treatment of DFAT cells with the adipogenic cocktail, and the downregulation of RhoA-ROCK signalling then promotes adipocyte differentiation via modulation of remodelling of the actin cytoskeleton. In addition, we found that treatment with LatA alone or Y-27632 alone, by inducing remodelling of the actin cytoskeleton, is sufficient to trigger adipocyte differentiation in the absence of an adipogenic cocktail. Our findings, combined with previous observations, thus suggest that a standard adipogenic cocktail directly elicits reorganization of the actin cytoskeleton through downregulation of RhoA-ROCK signalling, and that the disruption of actin stress fibres then acts as a trigger for the adipocytic differentiation of preadipocytes in vitro.

In differentiated adipocytes, cortical F-actin has an important regulatory role in the insulin-stimulated translocation of the glucose transporter GLUT4 (encoded by Slc2a4) from intracellular storage sites to the plasma membrane ${ }^{10}$, with the formation of cortical F-actin thus likely being a key event in adipogenesis. 
a

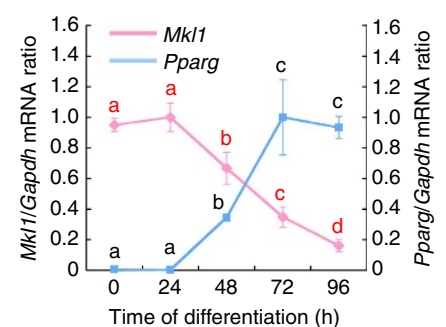

b
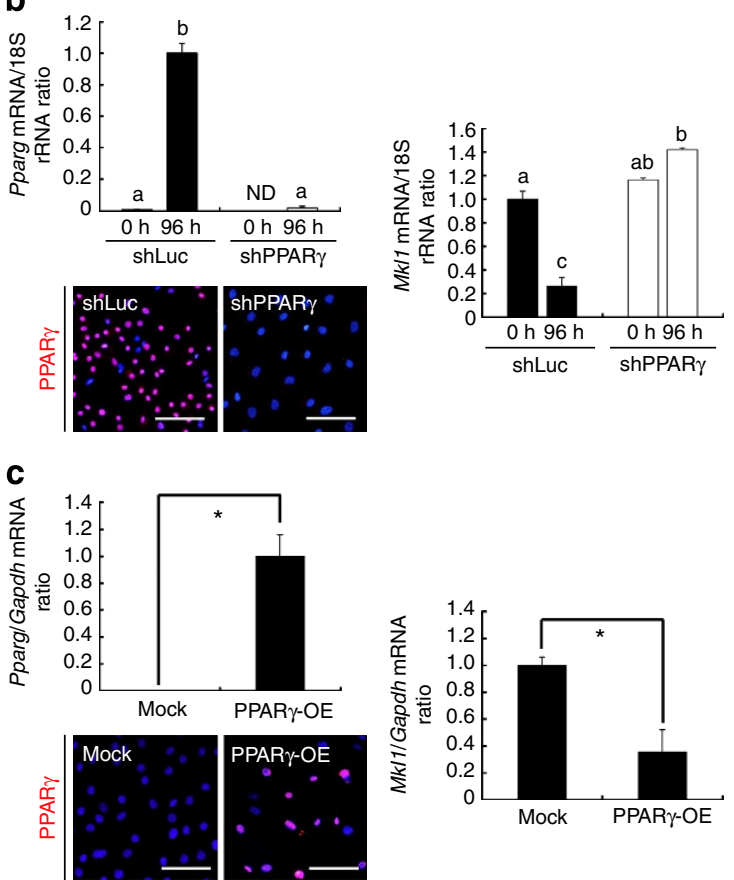

d

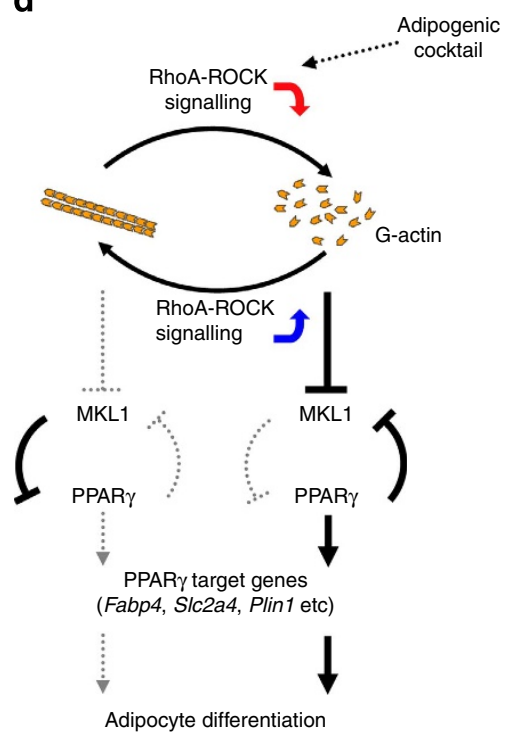

Figure 5 | PPAR $\gamma$ contributes to Mkl1 downregulation during adipocyte differentiation. (a) The relative abundance of Mk/1 and Pparg mRNAs at the indicated times after exposure of DFAT cells to inducers of adipocytic differentiation was also determined. (a-c) $P<0.05$, (a-d) $P<0.05$, Tukey's honest significant difference test. (b) DFAT cells stably expressing PPAR $\gamma$ (shPPAR $\gamma$ ) or luciferase (shLuc, control) shRNAs were exposed to inducers of adipogenesis for 0 or $96 \mathrm{~h}$ and then assayed for the relative abundance of Pparg and Mkl1 mRNAs. The cells were also subjected to immunofluorescence analysis of PPAR $\gamma$ after $96 \mathrm{~h}$. Nuclei were stained with Hoechst 33342. Scale bars, $100 \mu \mathrm{m}$. ND, not detected. (c) DFAT cells stably overexpressing PPAR $\gamma$ (PPAR $\gamma$-OE) or infected with the corresponding empty vector (Mock) were assayed for the relative abundance of Pparg and Mkl1 mRNAs. ${ }^{*} P<0.05$, Student's $t$-test. They were also subjected to immunofluorescence analysis of PPAR $\gamma$ expression. Nuclei were stained with Hoechst 33342. Scale bars, $100 \mu \mathrm{m}$. All quantitative data are means \pm s.d. ( $n=3$ experiments). (d) Model for the regulation of adipocyte differentiation. Regulation of MKL1 by disruption of actin stress fibres drives adipocyte differentiation.

In the present study, F-actin stress fibres were depolymerized to G-actin within $24 \mathrm{~h}$ after the induction of adipocyte differentiation, and the actin cytoskeleton was subsequently reorganized to form cortical F-actin structures within $48 \mathrm{~h}$. The abundance of transcripts for MKL1, which binds to G-actin, began to decline within $48 \mathrm{~h}$ after induction of adipocyte differentiation, and this downregulation of $M k l 1$ was controlled by PPAR $\gamma$. We also found that cells depleted of PPAR $\gamma$ maintained a high level of $M k l 1$ expression even after induction of adipocyte differentiation, and that these cells manifested disruption of actin stress fibres at $24 \mathrm{~h}$ but not the formation of cortical F-actin structures even at $96 \mathrm{~h}$ (Supplementary Fig. 4e). Together, these observations suggest that the downregulation of Mkl1 expression not only contributes to the continued activation of PPAR $\gamma$ but also promotes the organization of adipocytespecific cortical F-actin structures as a result of the release of $\mathrm{G}$-actin from MKL1-G-actin complexes.

Our findings provide new insight into the regulatory mechanism of adipocyte differentiation, which is shown to be triggered through regulation of MKL1 resulting from disruption of actin stress fibres. They also identify MKL1 as a novel gatekeeper in the regulation of adipogenesis as well as provide a basis for further studies of the relation between the dynamics of cell shape and transcription factor function during cellular differentiation. Lastly, given that activating mutations of RAC protein, which belongs to the RHO family of small GTPases and orchestrates actin polymerization, were found in a wide variety of human cancers and given their marked transforming ability ${ }^{38}$, we assume that aberrant differentiation associated with actin cytoskeleton dynamics can be a critical factor of tumorigenesis, and our works thus would potentially contribute to understanding cancer biology.

\section{Methods}

Cell culture. The mouse preadipocyte cell line DFAT was established from dedifferentiated mature adipocytes of GFP transgenic mice by ceiling culture (a method to culture lipid-containing adipocytes based on their lipid buoyancy) ${ }^{12}$. The mouse embryonic preadipocyte cell line 3T3-L1 and the mouse embryonic fibroblast cell line NIH 3T3 were obtained from the Japanese Collection of Research Bioresources (Tokyo, Japan). DFAT, 3T3-L1, or NIH 3T3 cells were cultured under a humidified atmosphere of $5 \% \mathrm{CO}_{2}$ and $95 \%$ air at $37^{\circ} \mathrm{C}$ on glass 
coverslips (Matsunami, Osaka, Japan) placed in tissue culture dishes (Falcon 3001; BD, Bedford, MA) containing Dulbecco's modified Eagle's medium (DMEM) (Nissui Pharmaceutical, Tokyo, Japan) supplemented with $10 \%$ fetal bovine serum (FBS) (Moregate BioTech, Bulimba, Queensland, Australia). DFAT or 3T3-L1 cells were grown to semiconfluence before induction of differentiation by exposure to DMEM supplemented with FBS (1\% or 10\%, respectively), $0.5 \mathrm{mM}$ IBMX (Wako, Osaka, Japan), $0.1 \mu \mathrm{M}$ dexamethasone (Wako), and insulin-transferrin-selenium-X supplement (final insulin concentration of $5 \mu \mathrm{g} \mathrm{ml}^{-1}$ ) (Invitrogen, Carlsbad, CA).

Time-lapse imaging. For time-lapse observation of actin dynamics during adipocyte differentiation, DFAT cells were infected with Cellular Lights Actin-RFP baculovirus expression vectors (Invitrogen) and then cultured in a glass-bottomed dish (Iwaki, Chiba, Japan). After the cells achieved semiconfluence, adipocyte differentiation was induced as described above and time-lapse fluorescence and differential interference contrast video microscopy was performed with the use of a microscope (Olympus LCV110) equipped with an incubation chamber. Images were acquired every $30 \mathrm{~min}$ with the use of a charge-coupled device camera (Retiga Exi, QImaging) equipped with a U Plan Super Apochromatic $\times 40,0.95$ numerical aperture objective and were analysed with MetaMorph software (Molecular Devices, Sunnyvale, CA).

RT and real-time PCR analysis. Total RNA was isolated from cells with the use of the Trizol reagent (Invitrogen), and portions $(1 \mu \mathrm{g})$ of the DNase I-treated RNA were subjected to reverse transcription (RT) with the use of high-capacity RNA-to-cDNA master mix (Applied Biosystems, Foster City, CA). The probes for PPAR $\gamma$ (Pparg; GenBank accession no. NM_011146.3; Mm00440940_m1), C/EBP $\alpha$ (Cebpa; GenBank accession no. NM_007678.3; Mm00514283_s1), fatty acid-binding protein 4 (Fabp4; GenBank accession no. NM_024406.2; Mm00445878_m1), GLUT4 (Slc2a4; GenBank accession no. NM_009204.2; Mm01245502_m1), perilipin 1 (Plin1; GenBank accession no. NM_175640.2; Mm00558672_m1) and MKL1 (Mkl1; GenBank accession no. NM_153049.2; Mm00461840_m1) genes were obtained fromTaqMan Pre-Developed Assay Reagents (Applied Biosystems). A mouse glyceraldehyde-3phosphate dehydrogenase (Gapdh; GenBank accession no. NM_008084.2) TaqMan probe (4352339E, Applied Biosystems) or eukaryotic 18S rRNA (GenBank accession no. X03205.1) TaqMan probe (4319413E, Applied Biosystems) was included as an endogenous control. The RT products $(2 \mu \mathrm{l})$ were subjected to real-time PCR analysis in a final volume of $10 \mu \mathrm{l}$ with the use of TaqMan Fast Universal PCR master mix (Applied Biosystems) and with an ABI7500 thermocycler. Each sample was assayed in triplicate.

Immunoblot analysis. Cells were washed extensively with phosphate-buffered saline (PBS) and then either recovered directly with a rubber scraper in SDS sample buffer (62.5 mM Tris-HCl (pH 6.8), 10\% SDS, 5\% glycerol, 5\% $\beta$-mercaptoethanol, $10 \%$ bromophenol blue) or subjected to extraction of nuclear and cytoplasmic fractions with the use of an NE-PER nuclear and cytoplasmic extraction reagent kit (Pierce, Rockford, IL). Samples were subjected to SDS-polyacrylamide gel electrophoresis, the separated proteins were transferred electrophoretically to a polyvinylidene difluoride membrane (Millipore, Bedford, MA), and nonspecific sites of the membrane were then blocked by incubation with Blocking One (Nacalai Tesque, Kyoto, Japan) for $1 \mathrm{~h}$ at room temperature. The membrane was then exposed for $24 \mathrm{~h}$ at $4{ }^{\circ} \mathrm{C}$ to rabbit polyclonal antibodies to PPAR $\gamma$ (1:500 dilution; P0744; Sigma-Aldrich, St Louis, MO), to lamin A/C (1:500 dilution; \#2032; Cell Signalling Technology, Beverly, MA), or to MEK1/2 (1:500 dilution; \#9122; Cell Signalling Technology); rabbit monoclonal antibodies to cofilin (1:1,000 dilution \#5175; Cell Signalling Technology); or mouse monoclonal antibodies to FLAG (1:1,000 dilution; F1804; Sigma-Aldrich), to GAPDH (1:10,000 dilution; G8795; Sigma-Aldrich), or to $\alpha$-tubulin (1:10,000 dilution; T5168; Sigma-Aldrich). The membrane was washed with PBS containing $0.02 \%$ Tween 20 , incubated for $1 \mathrm{~h}$ at room temperature with horseradish peroxidase-conjugated goat antibodies to rabbit or mouse immunoglobulin G (1:2,000 dilution; GE Healthcare, Tokyo, Japan), and washed again with PBS containing $0.02 \%$ Tween 20, after which immunoreactive proteins were visualized with the use of enhanced chemiluminescence reagents (GE Healthcare). Uncropped scans of the most important western blots were shown in Supplementary Fig. 7.

Immunofluorescence staining. Cells were fixed overnight at $4^{\circ} \mathrm{C}$ in zinc fixative solution (0.1 M Tris- $\mathrm{HCl}(\mathrm{pH} 7.4)$, calcium acetate $\left(475 \mu \mathrm{g} \mathrm{ml}^{-1}\right)$, zinc acetate $\left(5 \mathrm{mg} \mathrm{ml}^{-1}\right)$ and $\mathrm{ZnCl}_{2}\left(5 \mathrm{mg} \mathrm{ml}^{-1}\right)$ in ultrapure water) containing $0.2 \%$ Triton $\mathrm{X}-100$. They were then washed in Tris-buffered saline (TBS) and incubated for $1 \mathrm{~h}$ at room temperature with $10 \%$ normal goat or horse serum (Vector Laboratories, Burlingame, CA) and $1 \%$ bovine serum albumin (BSA) (Sigma-Aldrich) in TBS to block nonspecific binding of antibodies before staining with primary antibodies according to standard procedures. Primary antibodies included rabbit monoclonal antibodies to PPAR $\gamma$ (1:200 dilution; \#2435; Cell Signalling Technology); rabbit polyclonal antibodies to perilipin A/B (1:1,000 dilution; P1873; Sigma-Aldrich) and to MKL1 (1:100 dilution; ab49311; Abcam, Cambridge, MA); and mouse monoclonal antibodies to $\beta$-actin (1:1,000 dilution; A5441; Sigma-Aldrich), to HA (1:500 dilution; 1583816; Roche, Mannheim, Germany), and to FLAG (1:1,000 dilution; F1804; Sigma-Aldrich). Immune complexes were detected with Alexa Fluor 488- or Alexa Fluor 594-conjugated goat antibodies to rabbit or mouse immunoglobulin G (each at 1:2,000 dilution; Molecular Probes, Eugene, OR). Antibodies were diluted in TBS containing 1\% BSA. F-actin was stained with Alexa Fluor 488- or Alex Fluor 594-labelled phalloidin (Molecular Probes) at $200 \mathrm{U} \mathrm{ml}^{-1}$ in TBS, and G-actin was stained with Alexa Fluor 594-labelled DNase I (Molecular Probes) at $9 \mu \mathrm{g} \mathrm{ml}^{-1}$ in TBS. Cells were counterstained with Hoechst 33342 (Sigma-Aldrich) at $5 \mu \mathrm{g} \mathrm{ml}^{-1}$ in TBS and were observed with a laserscanning confocal microscope (Olympus FV-750) and a BIOREVO BZ-9000 fluorescence microscope (Keyence, Osaka, Japan).

Lipid staining. Lipid accumulation in adipocytes was detected by staining with oil red O (Wako). Cells were washed three times with PBS, fixed for $1 \mathrm{~h}$ at room temperature with $10 \%$ formalin in phosphate buffer, washed again with PBS and stained for $15 \mathrm{~min}$ at room temperature with a filtered solution of oil red $\mathrm{O}(0.5 \mathrm{~g}$ in $100 \mathrm{ml}$ of isopropyl alcohol). The cells were then washed twice with distilled water for $15 \mathrm{~min}$. For quantitation of lipid accumulation, dimethyl sulfoxide (DMSO) (500 $\mu \mathrm{l}$ per $35-\mathrm{mm}$ dish) was added to the washed and dried cells for $1 \mathrm{~min}$, after which the absorbance of the extracted dye at $510 \mathrm{~nm}$ was measured with a spectrophotometer (ND-1000; Nanodrop Technologies, Wilmington, DE) and was normalized by dish area.

Pharmacological agents. Cells were exposed to the following agents: $5 \mu \mathrm{M}$ phalloidin oleate (Calbiochem, San Diego, CA) dissolved in DMSO, $30 \mu \mathrm{M}$ Y-27632 (Calbiochem) dissolved in water, $0.2 \mu \mathrm{M}$ cytochalasin D (CytD) (Sigma-Aldrich) dissolved in DMSO, $0.4 \mu \mathrm{M}$ latrunculin A (LatA) (Calbiochem) dissolved in DMSO, $0.1 \mu \mathrm{M}$ swinholide A (SwinA) (Calbiochem) dissolved in ethanol or $1 \mu \mathrm{M}$ 4-hydroxytamoxifen (TAM) (Sigma-Aldrich) dissolved in ethanol.

Rho activation assay. Cells were lysed by incubation with a magnesiumcontaining buffer ( $25 \mathrm{mM}$ HEPES- $\mathrm{NaOH}$ (pH 7.5), $150 \mathrm{mM} \mathrm{NaCl}, 1 \%$ Igepal CA-630 detergent, $10 \mathrm{mM} \mathrm{MgCl} 2,1 \mathrm{mM}$ EDTA, $2 \%$ glycerol) supplemented with $25 \mathrm{mM} \mathrm{NaF}, 1 \mathrm{mM} \mathrm{Na}_{3} \mathrm{VO}_{4}$, and a protease inhibitor cocktail (Nacalai Tesque). The lysates were centrifuged at $14,000 \times g$ for $5 \mathrm{~min}$ at $4{ }^{\circ} \mathrm{C}$, and the resulting supernatants were incubated for $45 \mathrm{~min}$ at $4{ }^{\circ} \mathrm{C}$ with $25 \mu \mathrm{g}$ of a glutathione S-transferase fusion protein of the Rho-binding domain (amino acids 7-89) of rhotekin that was bound to glutathione-Sepharose beads (Millipore). The beads were washed three times with the magnesium-containing buffer and then subjected to immunoblot analysis with mouse monoclonal antibodies to RhoA, $-\mathrm{B}$ and $-\mathrm{C}$ (1:250 dilution; \#05-778; Millipore). Whole-cell lysates were also subjected to immunoblot analysis with the antibodies to Rho and those to GAPDH (loading control).

Transplantation and histological evaluation. All animal studies were performed according to the NIH Guide for the Care and Use of Laboratory Animals. C57BL/6J mice were obtained from Oriental Yeast (Tokyo, Japan). DFAT cells $\left(1 \times 10^{5}\right)$ collected with the use of a cell scraper were injected subcutaneously with a syringe above the sternum of 10 female mice at 8 weeks of age. After 2 weeks, cells at the injection site were retrieved, fixed in paraformaldehyde and embedded in paraffin. For immunohistochemical analysis, tissue sections were depleted of paraffin, washed with TBS, and stained with the use of a Vectastain ABC kit (Vector Laboratories). Primary antibodies included rabbit polyclonal antibodies to GFP (1:100 dilution; sc-8334; Santa Cruz Biotechnology, Santa Cruz, CA), to FABP4 (1:1,000 dilution; ab13979; Abcam) and to perilipin A/B (1:1,000 dilution; P1873; Sigma-Aldrich). The sections were counterstained with hematoxylin (Wako).

Plasmid transfection. The plasmids pEF-BOS-HA-RhoAV14 and pEF-BOS-HA RhoAN19 (ref. 39) plasmids were transfected into DFAT cells for $24 \mathrm{~h}$ using the Fugene HD reagent (Roche).

RNAi. Expression of shRNAs was achieved with the retroviral expression vector pRePS (kindly provided by T. Hara), which also contains a puromycin resistance gene. The sequences of the sense oligonucleotides were $5^{\prime}$-GTTTGAGTTTG CTGTGAAG- $3^{\prime}$ for PPAR $\gamma$ shRNA ${ }^{40}$ and $5^{\prime}$-CGTACGCGGAATACTTCGA- ${ }^{\prime}$ for luciferase shRNA (nonspecific control). pReps vectors transfected into Plat-E packaging cells ${ }^{41}$ using Fugene HD (Roche). Medium was replaced once after $24 \mathrm{~h}$, and viral supernatants were collected and filtered with $0.45-\mu \mathrm{m}$ cellulose acetate filters (Iwaki) $48 \mathrm{~h}$ after transfection. Retroviral infection was carried out in a sixwell plate for $48 \mathrm{~h}$, and infected cells were then subjected to selection in the presence of puromycin $\left(10 \mu \mathrm{g} \mathrm{ml}^{-1}\right)$. The sequences of siRNAs targeting cofilin 1 were $5^{\prime}$-GAUGAACACCAGGUCCUCCUU- $3^{\prime}$ for C $f l 1$-a, $5^{\prime}$-AAGAUCAAAAG CAGUUUGGGA- $3^{\prime}$ for $C f l 1$-b, and $5^{\prime}$-UCACUAUUGUGGUUAGAAGUU- $3^{\prime}$ for Cfll-c, and those of siRNAs targeting MKL1 were 5'-CGAGGACUAUUUGAAA CGGAA- $3^{\prime}$ for $M k l 1$-a and $5^{\prime}$-CCCACUCAGGUUCUUUCUCAA- $3^{\prime}$ for $M k l 1-\mathrm{b}$. The control siRNA sequence was $5^{\prime}$-GCGCGCUUUGUAGGAUUCG-3'. Cells 
were transfected with siRNA duplexes for $48 \mathrm{~h}$ with the use of Lipofectamine RNAiMAX (Invitrogen)

Retroviral gene transfer. The coding region for mCherry cDNA was amplified from the vector containing mCherry-MKlp2 (ref. 42) by PCR with the primers $5^{\prime}$-CCGCTCGAGATGGTGAGCAAGGGCGAGGAGGATAACATG-3' (XhoI_mCherry forward primer) and $5^{\prime}$-CGGAATTCTTCTTGTACAGCT CGTCCATGCCGCCGGTGG-3' (mCherry_EcoRI reverse primer), and the coding region for human ER was amplified from the pMV7-MycER plasmid ${ }^{43}$ by PCR with the primers $5^{\prime}$-CCGCTCGAGTCTGCTGGAGACATGAGAGCTGCCAA CCTT- $3^{\prime}$ (XhoI_ER forward primer) and $5^{\prime}$-CGGAATTCTTGACCGTGGCAG GGAAACCCTCTGCCTCCCC-3' (ER_EcoRI reverse primer), digested with XhoI and EcoRI, and ligated into the XhoI and EcoRI sites of pMXs- $3 \times$ FLAG-IP ${ }^{42}$. Human cDNAs for full-length MKL1 and MKL1-N100 were obtained by digestion of Addgene Plasmids (\#11978, \#27176) ${ }^{32}$ with EcoRI and BamHI, and the resulting fragments were cloned into the EcoRI and BamHI sites of pMXs- $3 \times$ FLAGmCherry-IP and/or pMXs- $3 \times$ FLAG-ER-IP. The resulting vectors were designated pMXs-3 × FLAG-mCherry-MKL1-IP, pMXs-3 × FLAG-ER-MKL1-IP, or pMXs-3 $\times$ FLAG-ER-MKL1-N100-IP, and pMXs-3 $\times$ FLAG-mCherry-IP or pMXs-3 $\times$ FLAG-ER-IP was used as a control. Mouse PPAR $\gamma$ cDNA (ImaGenes, Berlin, Germany) was cloned into the retroviral plasmid pMXs-puro (kindly provided by T. Kitamura). pMXs vectors were transfected into Plat-E packaging cells ${ }^{44}$ using FugeneHD (Roche). Medium was replaced once after $24 \mathrm{~h}$, and viral supernatants were collected and filtered with $0.45-\mu \mathrm{m}$ cellulose acetate filters (Iwaki) $48 \mathrm{~h}$ after transfection. Retroviral infection was carried out in a six-well plate for $48 \mathrm{~h}$, and infected cells were subjected to selection in the presence of puromycin $\left(10 \mu \mathrm{g} \mathrm{ml}^{-1}\right)$.

Microarray analysis. The quality of RNA was assessed using the Agilent 2100 Bioanalyzer (Agilent Technologies, Waldbronn, Germany). The RNA samples were labelled using the GeneChip One-Cycle Target Labeling and Control Reagent pakage (Affymetrix, Santa Clara, CA) and then hybridized to the Affymetrix GeneChip mouse genome 4302.0 array according to the manufacturer's instruction. Fluorescent images were visualized using a GeneChip Scanner 3000 (Affymetrix). Expression and raw expression data (CEL files) were summarized and normalized using the Robust Multi-array Average algorithm and the Bioconductor package affy (http://www.bioconductor.org/packages/2.0/bioc/html/affy.html). The Spotfire DecisionSite for Functional Genomics software package (TIBCO Software, Palo Alto, CA) was used for visualization of microarray data. Functional analyses were performed using DAVID Bioinformatics Resources 6.7 (http:// david.abcc.ncifcrf.gov/). Functional analysis identified the biological functions that were most significant to the data set.

Statistical analysis. Data are presented as means \pm s.d. and were analysed with Tukey's honest significant difference test or Student's $t$-test for comparisons between two or among three or more groups, respectively. A $P$-value of $<0.05$ was considered statistically significant.

\section{References}

1. Braun, T. \& Gautel, M. Transcriptional mechanisms regulating skeletal muscle differentiation, growth and homeostasis. Nat. Rev. Mol. Cell Biol. 12, 349-361 (2011).

2. de Crombrugghe, B., Lefebvre, V. \& Nakashima, K. Regulatory mechanisms in the pathways of cartilage and bone formation. Curr. Opin. Cell Biol. 13, 721-727 (2001)

3. Farmer, S. R. Transcriptional control of adipocyte formation. Cell Metab. 4, 263-273 (2006)

4. $\mathrm{Wu}, \mathrm{Z}$. et al. Cross-regulation of $\mathrm{C} / \mathrm{EBP} \alpha$ and $\operatorname{PPAR} \gamma$ controls the transcriptional pathway of adipogenesis and insulin sensitivity. Mol. Cell 3, 151-158 (1999).

5. Tontonoz, P., Hu, E. \& Spiegelman, B. M. Stimulation of adipogenesis in fibroblasts by PPAR $\gamma 2$, a lipid-activated transcription factor. Cell 79, 1147-1156 (1994)

6. Okuno, M., Arimoto, E., Nishizuka, M., Nishihara, T. \& Imagawa, M. Isolation of up- or down-regulated genes in PPAR $\gamma$-expressing NIH3T3 cells during differentiation into adipocytes. FEBS Lett. 519, 108-112 (2002).

7. Rosen, E. D. \& MacDougald, O. A. Adipocyte differentiation from the inside out. Nat. Rev. Mol. Cell Biol. 7, 885-896 (2006).

8. Smas, C. M. \& Sul, H. S. Control of adipocyte differentiation. Biochem. J. 309, 697-710 (1995)

9. Jaffe, A. B. \& Hall, A. Rho GTPases: biochemistry and biology. Annu. Rev. Cell Dev. Biol. 21, 247-269 (2005).

10. Kanzaki, M. \& Pessin, J. E. Insulin-stimulated GLUT4 translocation in adipocytes is dependent upon cortical actin remodeling. J. Biol. Chem. 276, 42436-42444 (2001)

11. Noguchi, M. et al. Genetic and pharmacological inhibition of Rho-associated kinase II enhances adipogenesis. J. Biol. Chem. 282, 29574-29583 (2007)
12. Nobusue, H., Endo, T. \& Kano, K. Establishment of a preadipocyte cell line derived from mature adipocytes of GFP transgenic mice and formation of adipose tissue. Cell Tissue Res. 332, 435-446 (2008).

13. Yagi, K., Kondo, D., Okazaki, Y. \& Kano, K. A novel preadipocyte cell line established from mouse adult mature adipocytes. Biochem. Biophys. Res. Commun. 321, 967-974 (2004).

14. Spiegelman, B. M. \& Ginty, C. A. Fibronectin modulation of cell shape and lipogenic gene expression in 3T3-adipocytes. Cell 35, 657-666 (1983).

15. McBeath, R., Pirone, D. M., Nelson, C. M., Bhadriraju, K. \& Chen, C. S. Cell shape, cytoskeletal tension, and RhoA regulate stem cell lineage commitment. Dev. Cell 6, 483-495 (2004).

16. Kilian, K. A., Bugarija, B., Lahn, B. T. \& Mrksich, M. Geometric cues for directing the differentiation of mesenchymal stem cells. Proc. Natl Acad. Sci. USA 107, 4872-4877 (2010).

17. Dupont, S. et al. Role of YAP/TAZ in mechanotransduction. Nature 474, 179-183 (2011).

18. Cristancho, A. G. \& Lazar, M. A. Forming functional fat: a growing understanding of adipocyte differentiation. Nat. Rev. Mol. Cell Biol. 12, 722-734 (2011)

19. Ridley, A. J. \& Hall, A. The small GTP-binding protein Rho regulates the assembly of focal adhesions and actin stress fibres in response to growth factors. Cell 70, 389-399 (1992).

20. Riento, K. \& Ridley, A. J. Rocks: multifunctional kinases in cell behaviour. Nat. Rev. Mol. Cell Biol. 4, 446-456 (2003).

21. Hamm, J. K., El Jack, A. K., Pilch, P. F. \& Farmer, S. R. Role of PPAR $\gamma$ in regulating adipocyte differentiation and insulin-responsive glucose uptake. Ann. NY Acad. Sci. 18, 134-145 (1999).

22. Tashiro, K. et al. Efficient adenovirus vector-mediated PPAR $\gamma$ gene transfer into mouse embryoid bodies promotes adipocyte differentiation. J. Gene Med 10, 498-507 (2008)

23. Tontonoz, P. \& Spiegelman, B. M. Fat and beyond: the diverse biology of PPAR $\gamma$. Annu. Rev. Biochem. 77, 289-312 (2008).

24. Qian, S. W. et al. Characterization of adipocyte differentiation from human mesenchymal stem cells in bone marrow. BMC Dev. Biol. 10, 47 (2010).

25. Bamburg, J. R. Proteins of the ADF/cofilin family: essential regulators of actin dynamics. Annu. Rev. Cell Dev. Biol. 15, 185-230 (1999).

26. Olson, E. N. \& Nordheim, A. Linking actin dynamics and gene transcription to drive cellular motile functions. Nat. Rev. Mol. Cell Biol. 11, 353-365 (2010).

27. Miralles, F., Posem, G., Zaromytidou, A. I. \& Treisman, R. Actin dynamics control SRF activity by regulation of its coactivator MAL. Cell 113, 329-342 (2003).

28. Vartiainen, M. K., Guettler, S., Larijiani, B. \& Treisman, R. Nuclear actin regulates dynamic subcellular localization and activity of the SRF cofactor MAL. Science 316, 1749-1752 (2007).

29. Yin, J. W. et al. Mediator MED23 plays opposing roles in directing smooth muscle cell and adipocyte differentiation. Genes Dev. 26, 2192-2205 (2012).

30. Lyubimova, A., Bershadsky, A. D. \& Ben-Ze'ev, A. Autoregulation of actin synthesis responds to monomeric actin levels. J. Cell Biochem. 65, 469-478 (1997).

31. Bubb, M. R., Spector, I., Bershadsky, A. D. \& Korn, E. D. Swinholide A is a microfilament disrupting marine toxin that stabilizes actin dimmers and severs actin filaments. J. Biol. Chem. 270, 3463-3466 (1995).

32. Cen, B. et al. Megakaryoblastic leukemia 1, a potent transcriptional coactivator for serum response factor (SRF), is required for serum induction of SRF target genes. Mol. Cell Biol. 23, 6597-6608 (2003)

33. Guettler, S., Vartiainen, M. K., Miralles, F., Larijani, B. \& Treisman, R. RPEL motifs link the serum response factor cofactor MAL but not myocardin to Rho signaling via actin binding. Mol. Cell Biol. 28, 732-742 (2008).

34. Sun, Y. et al. Acute myeloid leukemia-associated Mkl1 (Mrtf-a) is key regulator of mammary gland function. Mol. Cell Biol. 26, 5809-5826 (2006).

35. Liu, J. et al. Changes in integrin expression during adipocyte differentiation. Cell Metab. 2, 165-177 (2005).

36. Vassaux, G., Gaillard, D., Ailhaud, G. \& Négrel, R. Prostacyclin is a specific effector of adipose cell differentiation. Its dual role as a cAMP- and $\mathrm{Ca}^{2+}$. elevating agent. J. Biol. Chem. 267, 11092-11097 (1992).

37. Petersen, R. K. et al. Cyclic AMP (cAMP)-mediated stimulation of adipocyte differentiation requires the synergistic action of Epac- and cAMPdependent protein kinase-dependent processes. Mol. Cell Biol. 28, 3804-3816 (2008).

38. Kawazu, M. et al. Transforming mutations of RAC guanosine triphosphatases in human cancers. Proc. Natl Acad. Sci. USA 110, 3029-3034 (2013)

39. Amano, M. et al. Identification of a putative target for Rho as the serine-threonine kinase protein kinase N. Science 271, 648-650 (1996).

40. Shimizu, T. et al. c-MYC overexpression with loss of Ink4a/Arf transforms bone marrow stromal cells into osteosarcoma accompanied by loss of adipogenesis. Oncogene 29, 5687-5699 (2010). 
41. Fujino, R. S. et al. Spermatogonial cell-mediated activation of an I $\mathrm{I} B$ independent nuclear factor- $\mathrm{\kappa B}$ pathway in Sertoli cells induces transcription of the lipocalin-2 gene. Mol. Endocrinol. 20, 904-915 (2006).

42. Kitagawa, M., Fung, S. Y., Onishi, N., Saya, H. \& Lee, S. H. Targeting Aurora B to the equatorial cortex by MKlp2 is required for cytokinesis. PLoS One 8, e64826 (2013)

43. Eilers, M., Picard, D., Yamamoto, K. R. \& Bishop, J. M. Chimaeras of Myc oncoprotein and steroid receptors cause hormone-dependent transformation of cells. Nature 340, 66-68 (1989).

44. Morita, T., Kojima, T. \& Kitamura, T. Plat-E: an efficient and stable system for transient packaging of retroviruses. Gene Ther. 7, 1063-1066 (2000).

\section{Acknowledgements}

We thank T. Hara for the retroviral expression vector pRePS; T. Kitamura for the retroviral expression vector pMXs-puro; O. Sampetrean, S. Kuninaka, and H. Naoe for discussion; RM. Yonamine, X. Wang, H. Ito, N. Yanagihara, and N. Hirose for technical assistance; and K. Arai for secretarial assistance. We are grateful to the Collaborative Research Resources, School of Medicine, Keio University for technical support and reagents. This work was partly supported by a grant from the Ministry of Education, Science, Sports, and Culture of Japan (HS) and a grant from Okinawa Research and Industrialization for the Forefront Medical Care (KK).

\section{Author contributions}

H.N. performed most of the experimental works and wrote the manuscript. N.O. helped experimental design and contributed to the writing of the manuscript. T.S., E.S., Y.O., Y.S., T.C and K.A. helped with the experiments and to review the manuscript prior to submission. H.S. and K.K. supervised the project and manuscript editing, and prepared the manuscript.

\section{Additional information}

Accession codes: The raw and processed microarray data have been deposited in the Gene Expression Omnibus under the accession code GSE52334.

Supplementary Information accompanies this paper at http://www.nature.com/nature communications

Competing financial interests: The authors declare no competing financial interests.

Reprints and permission information is available online at http://npg.nature.com/ reprintsandpermissions/

How to cite this article: Nobusue, H. et al. Regulation of MKL1 via actin cytoskeleton dynamics drives adipocyte differentiation. Nat. Commun. 5:3368 doi: 10.1038/ ncomms4368 (2014). 